\title{
Sim Track User’s Manual (v 1.0)
}

\author{
Y. Luo
}

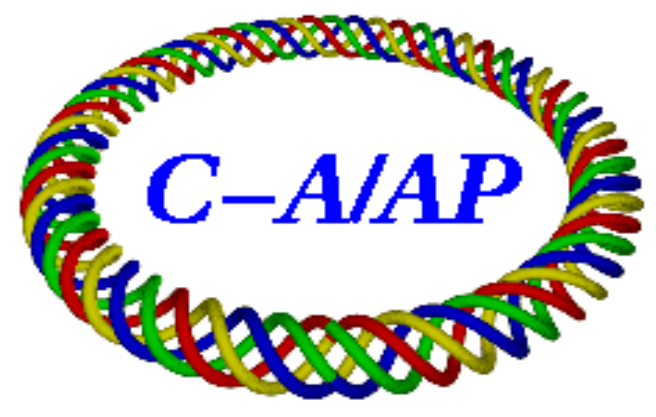

\section{Collider-Accelerator Department Brookhaven National Laboratory \\ Upton, NY 11973}

Notice: This document has been authorized by employees of Brookhaven Science Associates, LLC under Contract No. DE-AC02-98CH10886 with the U.S. Department of Energy. The United States Government retains a nonexclusive, paid-up, irrevocable, world-wide license to publish or reproduce the published form of this document, or allow others to do so, for United States Government purposes. 


\title{
SimTrack User's Manual ( v 1.0 )
}

\author{
Yun Luo \\ Brookhaven National Laboratory, Upton, NY 11973, USA
}

SimTrack is a simple c++ library designed for the numeric particle tracking in the high energy accelerators. It adopts the 4 th order symplectic integrator $[1,2]$ for the optical transport in the magnetic elements. The 4-D and 6-D weak-strong beam-beam treatments [3, 4] are integrated in it for the beam-beam studies. SimTrack is written with $\mathrm{c}++$ class and standard template library. It provides versatile functions to manage elements and lines. It supports a large range of types of elements. New type of element can be easily created in the library. SimTrack calculates Twiss, coupling and fits tunes, chromaticities and corrects closed orbits. AC dipole and AC multipole are available in this library. SimTrack allows change of element parameters during tracking.

\section{Get Started}

\subsection{Including simtrack.h}

SimTrack library is written in $\mathrm{C}++$. It currently has only one file simtrack.h with $6000+$ lines. To use SimTrack library in a general $\mathrm{C}++$ program, you simply need to include simtrack.h in the beginning of your source code. The syntax and functions of $\mathrm{C}++$ and SimTrack library will be applied to the source code.

As an example, following is a source code "test.cpp" which uses SimTrack library to calculate some accelerator physics parameters. In test.cpp, some SimTrack functions are used, such as Read_MADXLattice() to read in the lattice file generated from MADX, Get_Twiss() to calculate Twiss parameters, Get_Chrom() to calculate the chromaticities.

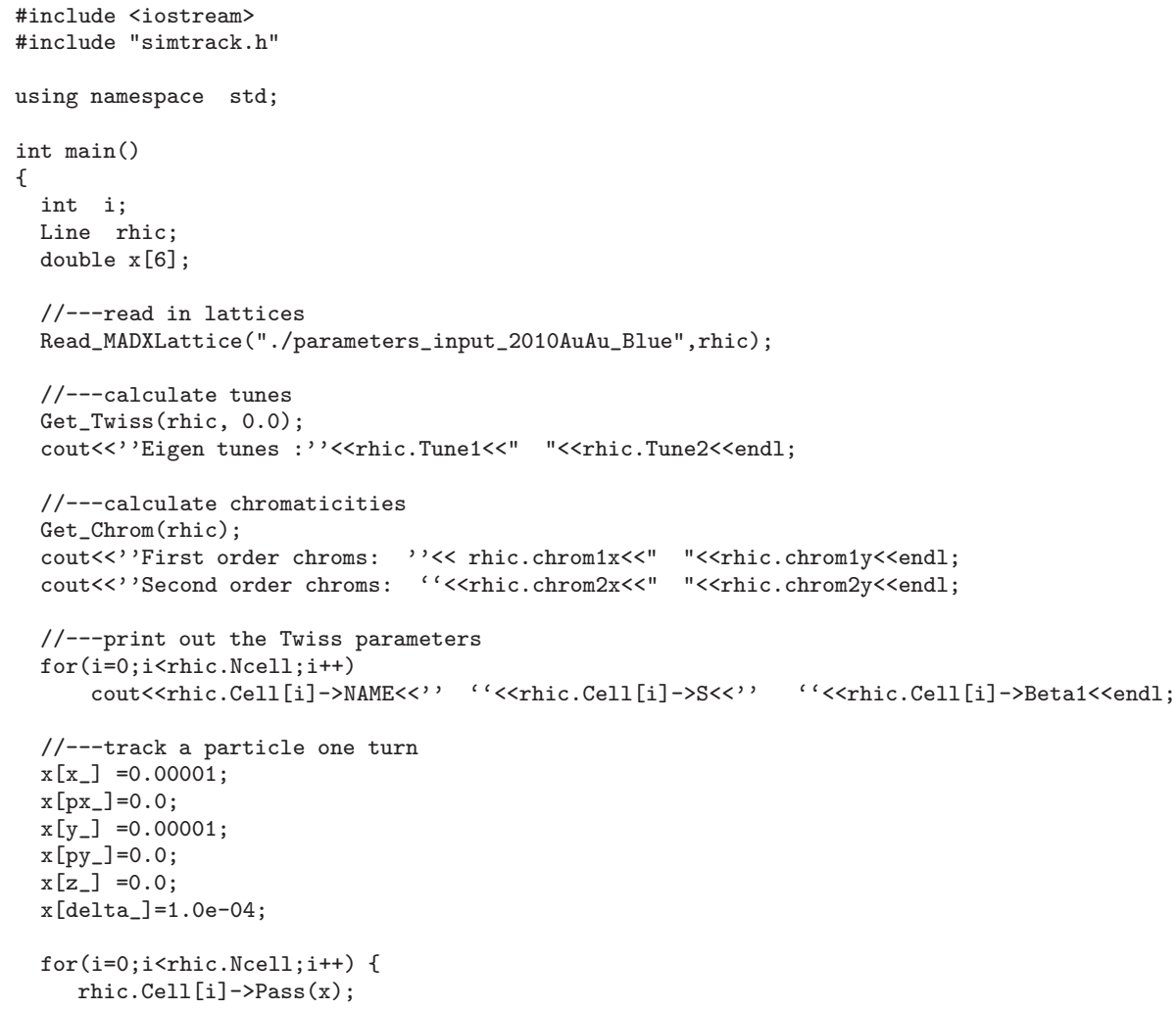




\subsection{Input file generated from MADX}

The lattice input file "parameters_input_2010AuAu_Blue" in the above code is generated from MADX. To generate it from MADX, we simply include the following block in the MADX input file and run MADX. The output file "parameters_input_2010AuAu_Blue" includes all the magnetic strengths of each element of the ring "rhic". Please pay attention that function Read_MADXLattice() may omit some types of elements and simply transfers them into "DRIFT" type. Function Read_MADXLattice() also zero the cavity voltage ("VRF") of RFCAV element and the bunch particle number("NP") of BEAMBEAM element and so on. Actually we can write interface functions in SimTrack to read in any format of lattice. SimTrack also can define and build up a ring or line from single element by using line manipulation functions to be discussed later.

use, period=rhic;

select, flag=twiss, column=NAME, KEYWORD, S, L, ANGLE, E1, E2, tilt, KOL, KOSL, K1L, K1SL, K2L, K2SL , K3L , K3SL , K4L , K4SL, K5L, K5SL, K6L , K6SL, K7L, K7SL, K8L, K8SL , K9L, K9SL, K10L, K10SL, KS ; twiss, table=twiss, file=pparameters_input_2010AuAu_Blue;

stop;

\subsection{Install GSL library}

SimTrack library uses GSL library to solve $4 \times 4$ eigen system to get eigen vectors and eigen tunes. Therefore, to compile source code using SimTrack library, the GSL library should be included too. If GSL library is not installed in your system by your system administer, you can download the source code from http://www.gnu.org/software/gsl/ and copy to a work directory. Under that work directory, follow the following commands to install it under your local directory $\$ H O M E /$ bin/GSL.

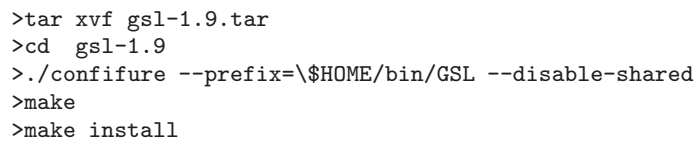

\subsection{Compiling source code}

For simplicity, the author created a short script called "MakeST" under $\$ H O M E /$ bin to facilitate the compiling source codes using SimTrack library. "MakeST" reads

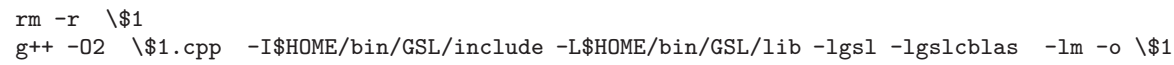

We can compile a source code, for example test.cpp, using SimTrack library under any directory by typing

$>$ MakeSimTrack test

If compiling is successful, you will have the executable file named "test". To run it, you simply type

$>. /$ test

\section{Element}

\subsection{Common parameters and functions of element}

SimTrack supports a large range of element types. Regarding the specific type of element, each element has the following common parameters and functions.

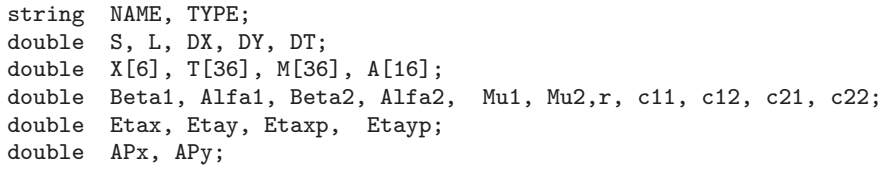




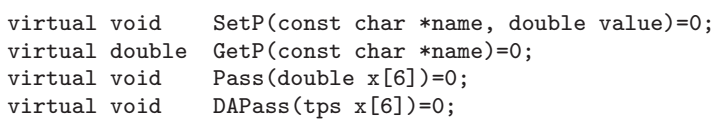

The definitions of the above parameters are:

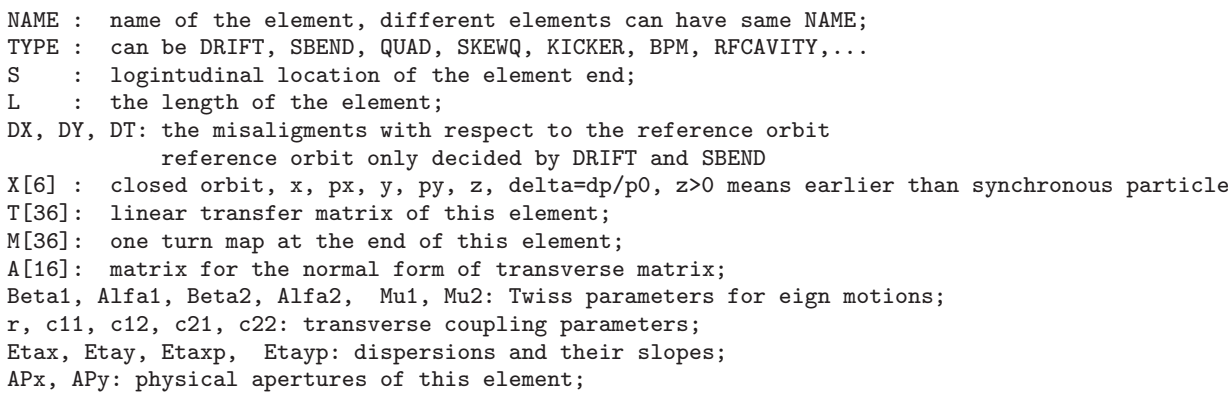

\subsection{Access to common parameters and functions}

To access the above common parameters and functions of element, we simply use pointers. For example, to get the Beta1 of an element in the line "rhic", we use

rhic.Cell[i]->Beta1

where $\mathrm{i}$ is the index of the elements in a ring or line, i starts from 0. For the line "rhic", there are rhic.Ncell elements. The index of the last element is (rhic.Ncell-1).

To transfer a particle with coordinate double $\mathrm{x}[6]$ through an element, we use

rhic.Cell[i]->Pass (x) .

Pass() and DAPass() only differ in the input types of data. Pass() is used for double $\mathrm{x}[6]$ while DAPass() is used to transfer the linear tpsa data. DAPass() is used during one-turn map and Twiss calculations.

\subsection{Specific types of element}

SimTrack supports the following types of element. Each type of element has its specific parameters. For example, QUAD element has parameter "K1L" while SEXT element has "K2L". These specific parameters only can be accessed and modified with functions $\operatorname{GetP}()$ and $\operatorname{SetP}()$. Following lists the accepted types of element and their specific parameters in SimTrack library.

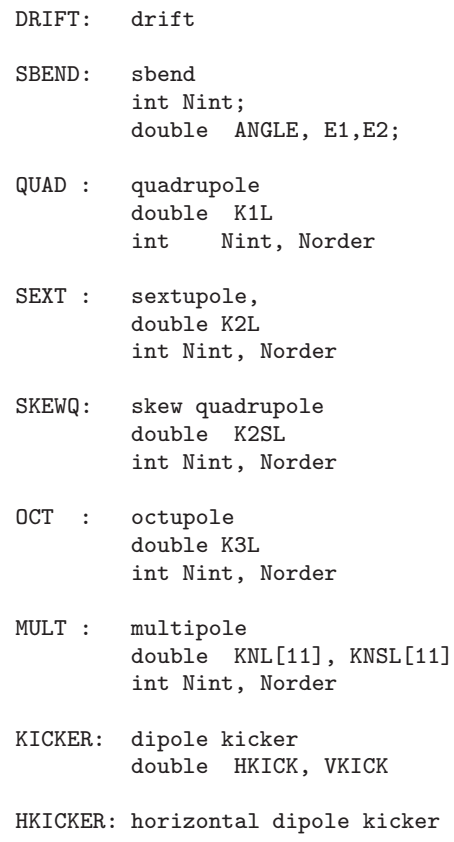

HKICKER: horizontal dipole kicker 
double HKICK

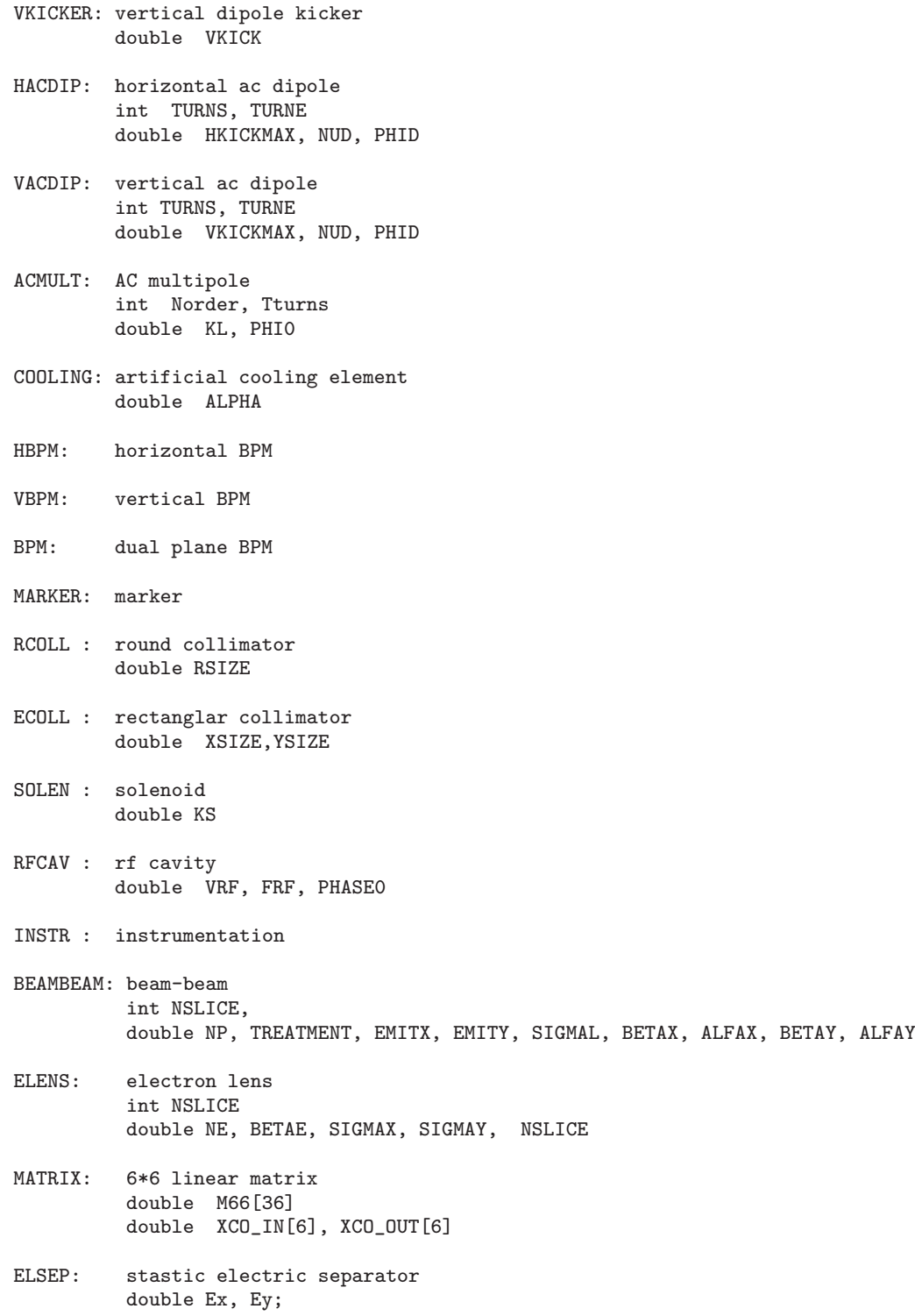

In the above, "Nint" and "Nslice" is the integration steps in the particle transferring. "Norder" is maximum order of the magnetic field. They are automatically set in the construction functions of magnetic elements. SimTrack uses 4 -th order symplectic integrator to track particles.

\subsection{Access to specific parameters}

As an example, the following block source code prints out the strengths of all quadrupoles in the line "rhic", where $\operatorname{GetP}()$ is used,

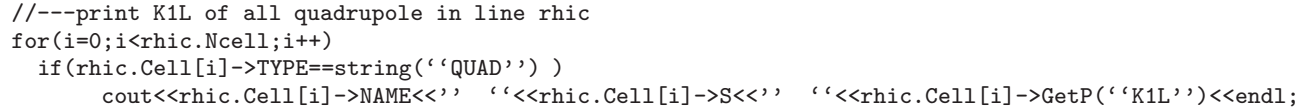

Another example to set all sextupole "SF" strength to be 0 ., where $\operatorname{SetP}()$ is used,

//---set SF strength to 0 .

for $(i=0 ; i<$ rhic.Ncell; $i++)$

if (rhic.Cell [i]->NAME==string ("SF' ') and rhic.Cell[i]->TYPE==string ("SEXT' ') )

rhic.Cell [i] ->SetP('K2L', , . );

Again, to access or set the specific parameters of one type of element, we need to use functions GetP() and $\operatorname{SetP}()$ of that type of element. However, to access the common paramters of an element, like Twiss parameters etc, we use pointers. 


\subsection{Create an element}

To create an element with a specific type in the source code, we use the construction function of that element type.

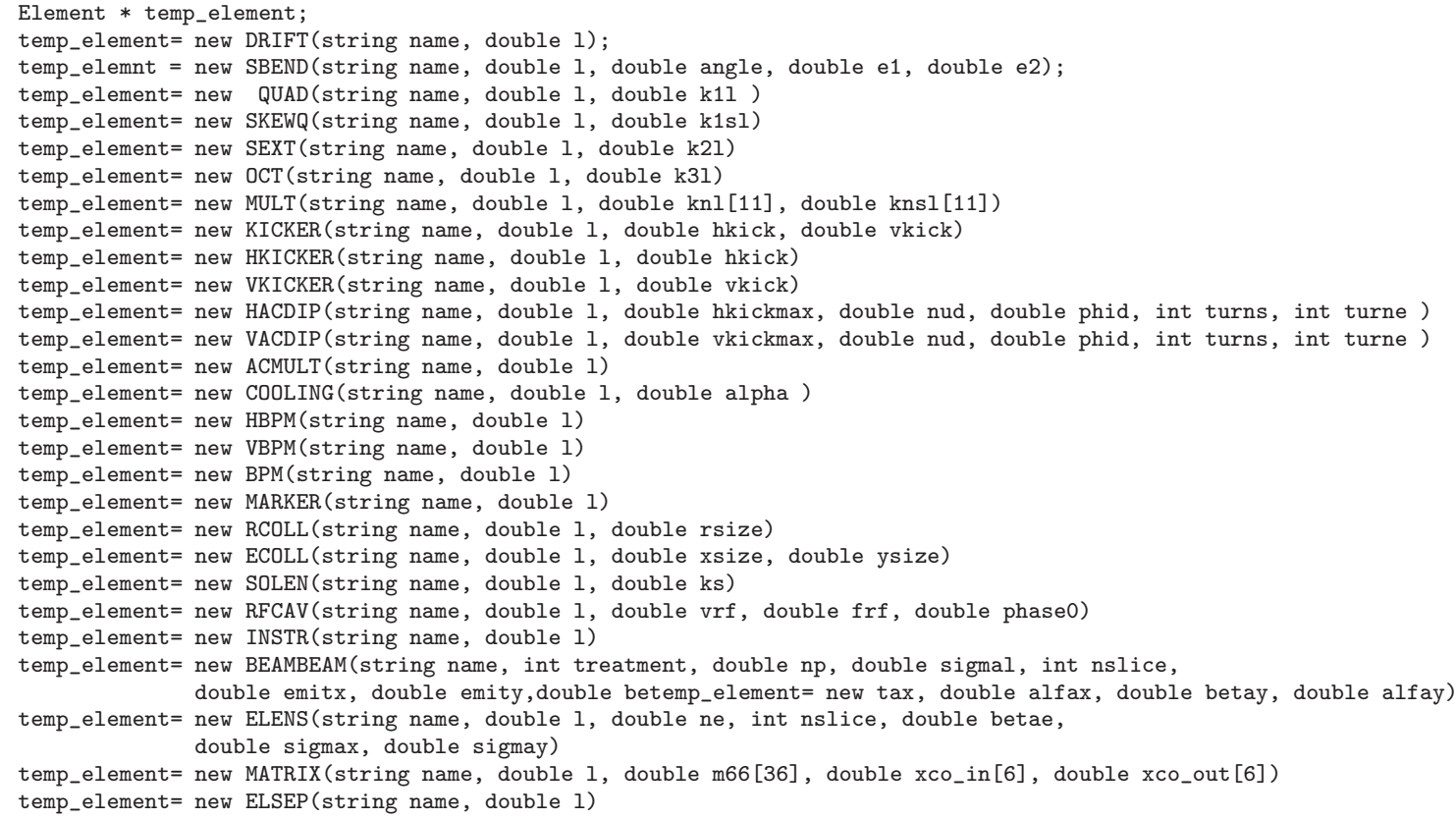

In SimTrack, BEAMBEAM type of element must have zero length.

\section{Line}

\subsection{Definitions}

Line is a sequence of specific elements. Line has the following parameters and functions,

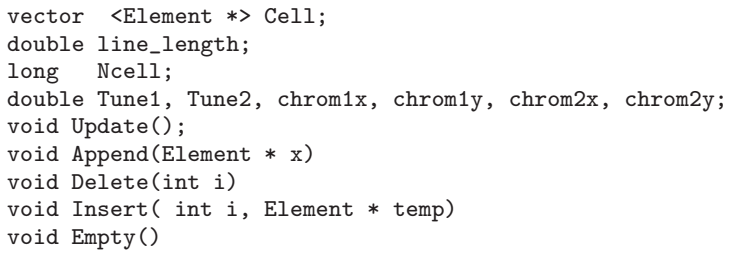

The definitions of Line's parameters are

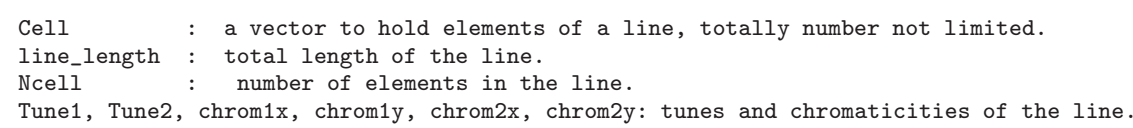

The definitions of line manipulation functions

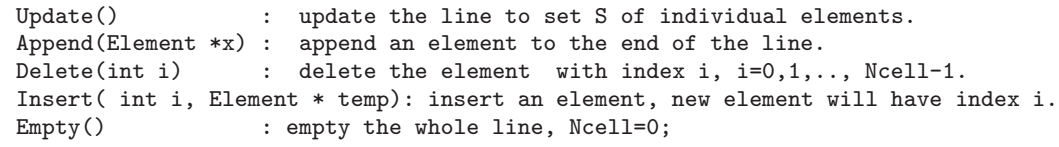

There are two line manipulation functions which are not the member functions of line.

void Rewind_Line(Line \& linename, int $\mathrm{k}$ )

void Inverse_Line(Line \& linename)

Rewind_Line() is to set the new starting point of a ring. The new starting element will be Cell[k] of previous line. Rewind_Line() doesn't change the order of elements. Inverse_Line() is to revert the order of elements of the line. Inverse_Line() doesn't change the strengths of elements. 


\subsection{Some examples}

As an example, following block assign voltage to an existing rf cavity element and delete, define, insert beam-beam elements in the line "rhic".

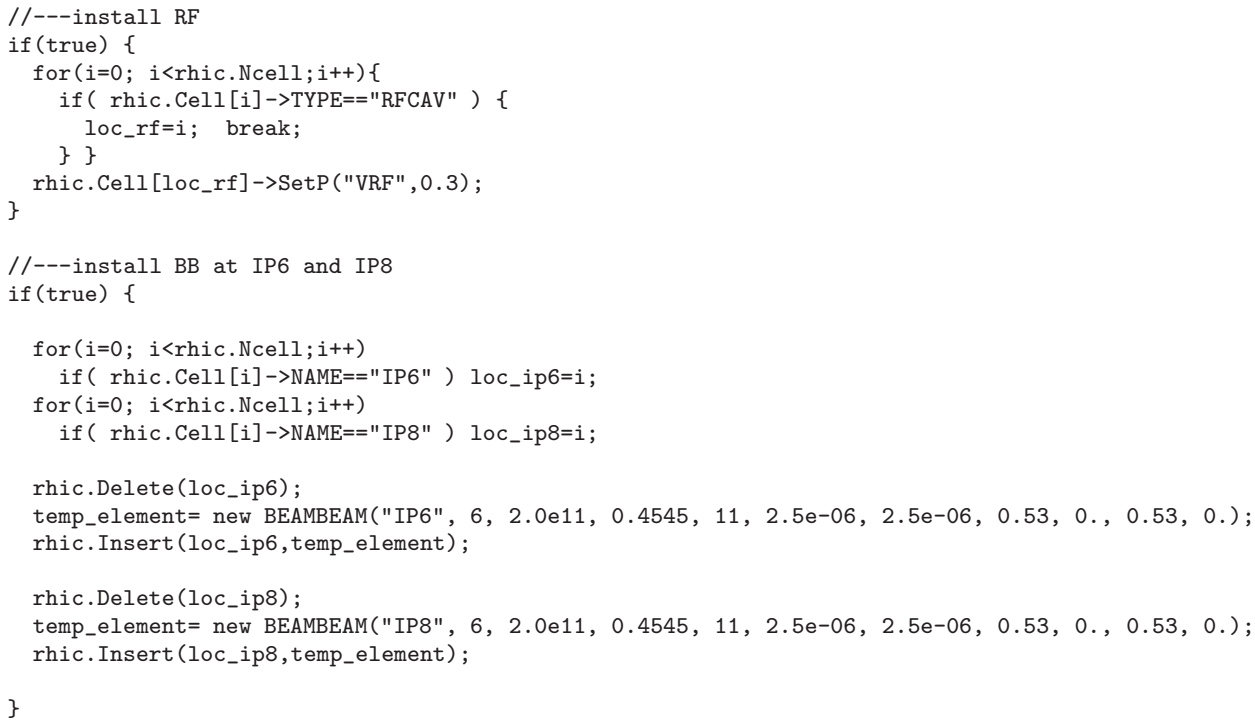

As another example, the following source code to revert the rhic Blue ring lattice to check the parameter changes.

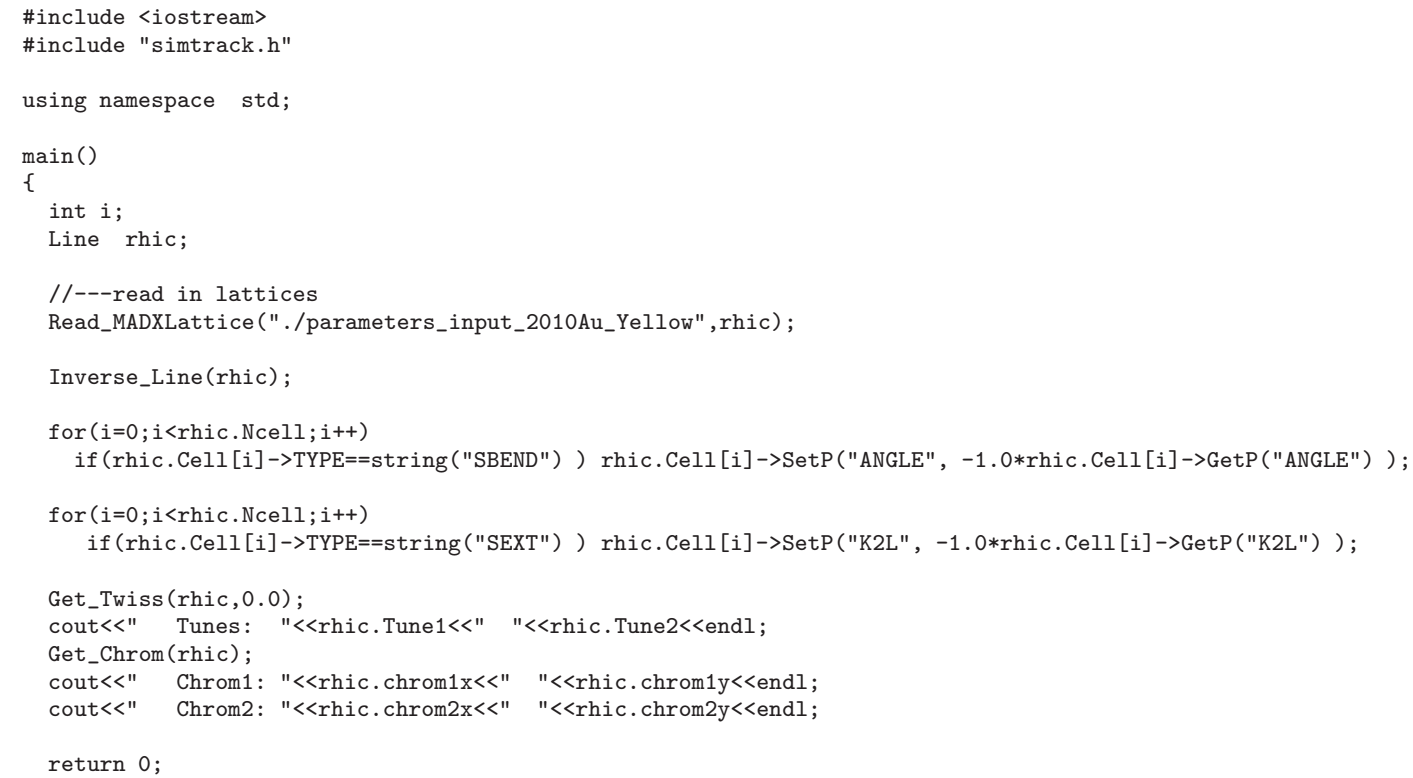

The following source code creates a simple ring and check its tunes.

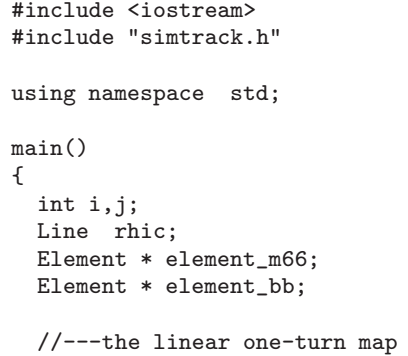




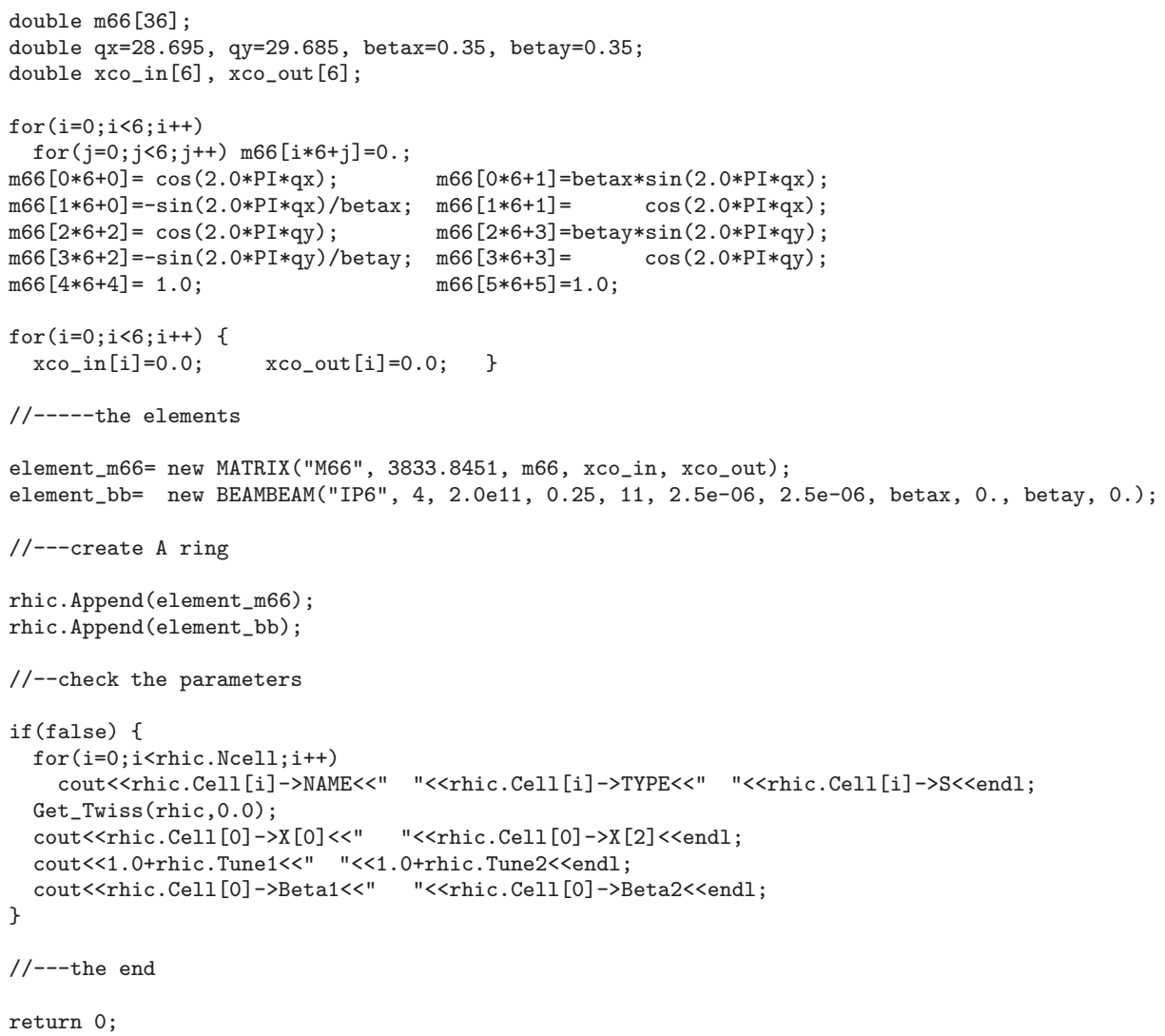

The following source code to insert an AC dipole into the ring and track a particle 10000 turns and print out turn-by-turn data.

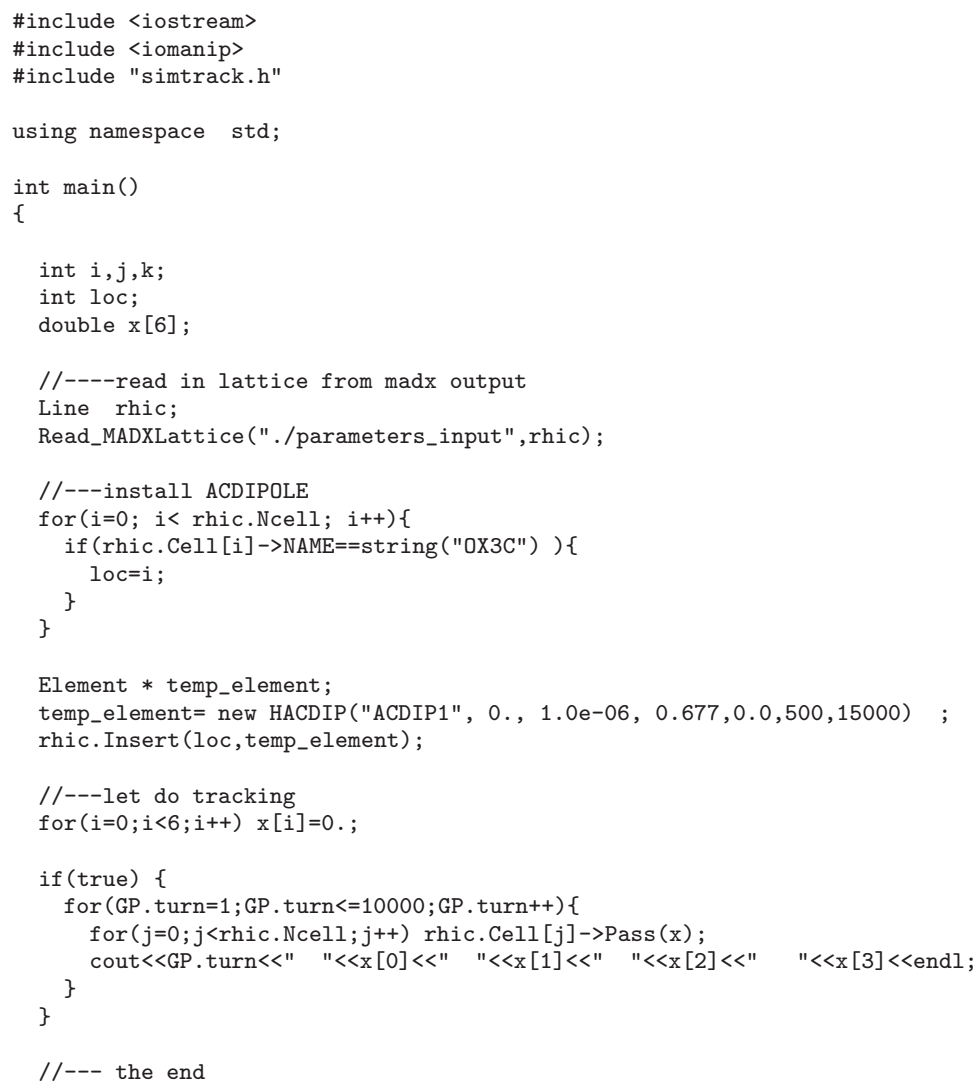




\subsection{Assumed global parameters}

The following are the default line parameters used in the SimTrack library. They are based on rhic $250 \mathrm{GeV}$ polarized proton run.

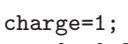

\section{Optics, Fitting and Closed Orbit Correction}

\subsection{Optics calculation}

SimTrack supplies general optical calculation functions, such as,

void Get_Orbit(Line \& linename, double deltap)

void Get_Twiss (Line \& linename, double deltap)

void Get_Chrom( Line \& linename)

void Get_Dispersion(Line \& linename, double deltap)

Coupling parameters are calculated with Get_Twiss(). And Get_Twiss() already includes Get_Orbit(). Get_Twiss() doesn't calculate dispersion. Dispersion is calculated with function Get_Dispersion(). Get_Dispersion() should be proceeded by Get_Twiss().

SimTrack supplies limited fitting functions, such as,

void Fit_Tune(Line \& linename, double q1, double q2, const char $*$ qf_name, const char * qd_name)

void Fit_Chrom(Line \& linename, double chrom1x_want, double chrom1y_want, const char $*$ sf_name, const char $*$ sd_name )

void Fit_Chrom_RHIC8fam(Line \& linename, double chrom1x_want, double chrom1y_want )

The following functions simplify the reading and setting strengths of a magnet family which share the same name. In SimTrack, the elements with same names can have different strengths in SimTrack, which is different from MADX.

double Get_KL (Line \& linename, const char * name, const char * kl)

void Set_KL (Line \& linename, const char $*$ name, const char $* \mathrm{kl}$, double strength)

void Set_dKL (Line \& linename, const char $*$ name, const char $* \mathrm{kl}$, double dstrength)

The following functions work one-turn and section maps. Most users don't need to know or use these functions. They are used during optics calculation. The Twiss and coupling parameters are calculated in SimTrack based on Ref. [5].

void Get_OneTurnMap(Line \& linename, double deltap)

void Get_ElementMap(Line \& linename, double deltap)

void Get_SectionMap(Line \& linename, int i1, int i2, double deltap, double t66[36] )

void Cal_A44 (Line \& linename, double deltap)

void Trace_A44(Line \& linename, double deltap)

As an example, let us look into function Fit_Chrom(),

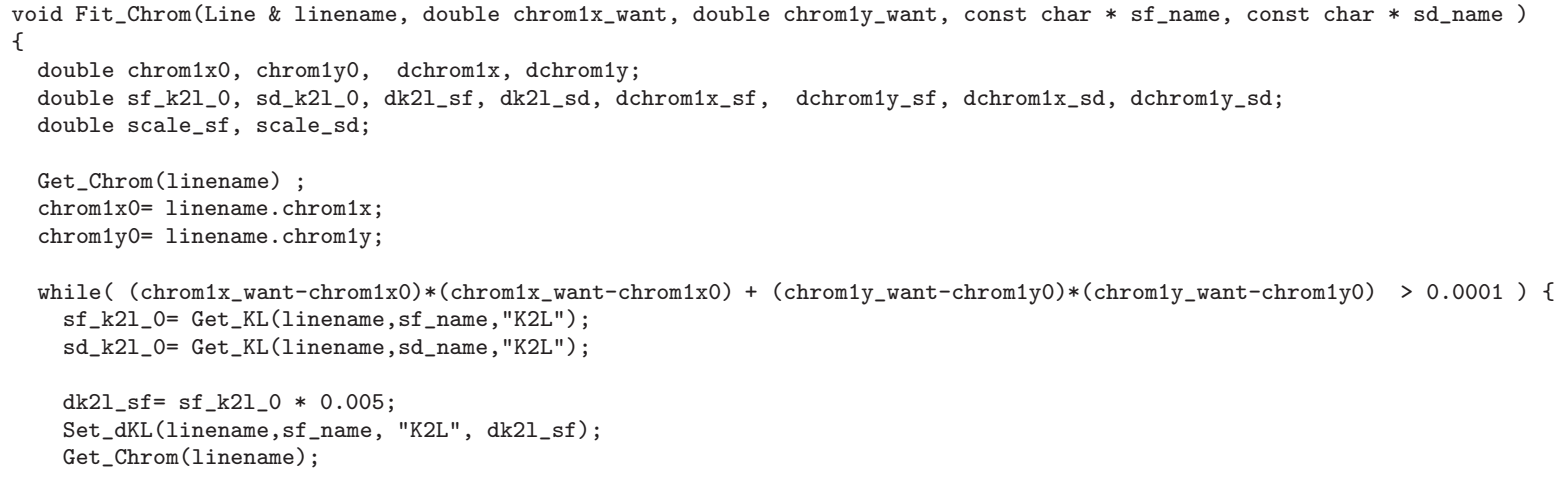




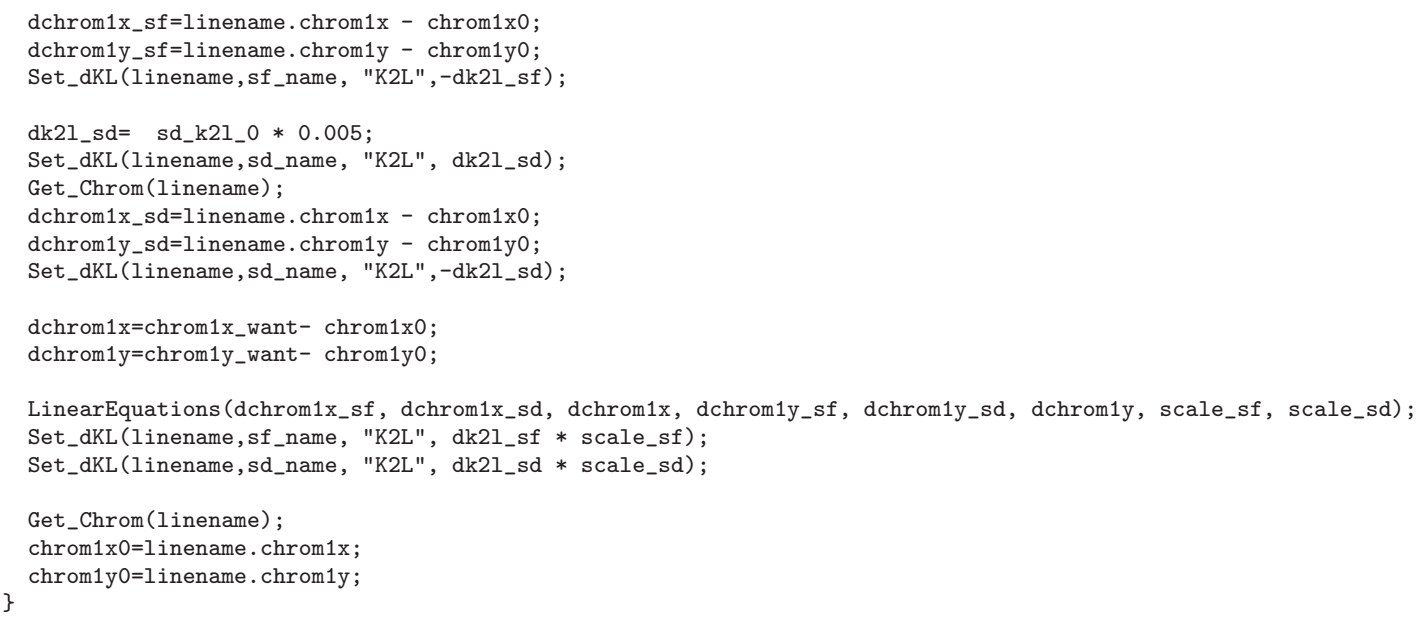

\subsection{Closed orbit correction}

SimTrack also supplies functions for closed orbit correction,

void Correct_Orbit_SVD(Line \& linename, int $m$, int $n$, vector<int> bpm_index, vector<int> kicker_index, int plane)

void Correct_Orbit_SlidingBump1(Line \& linename, int $\mathrm{m}$, int $\mathrm{n}$, vector<int> bpm_index, vector<int> kicker_index, int plane)

void Correct_Orbit_SlidingBump2(Line \& linename, int $\mathrm{m}$, int $\mathrm{n}$, vector<int> bpm_index, vector<int> kicker_index, int plane)

void Orbit_Status( Line linename, vector<int> bpm_index, int plane, double \&orbit_mean, double \& orbit_rms )

The input of orbit correction are vector containers of the index of BPMs and correctors. Flag plane $=0$ means horizontal orbit correction.

Following is an example to do horizontal closed orbit correction with all available horizontal BPMs and horizontal dipole correctors. The initial random closed orbit is generated with quadrupole horizontal misalignments.

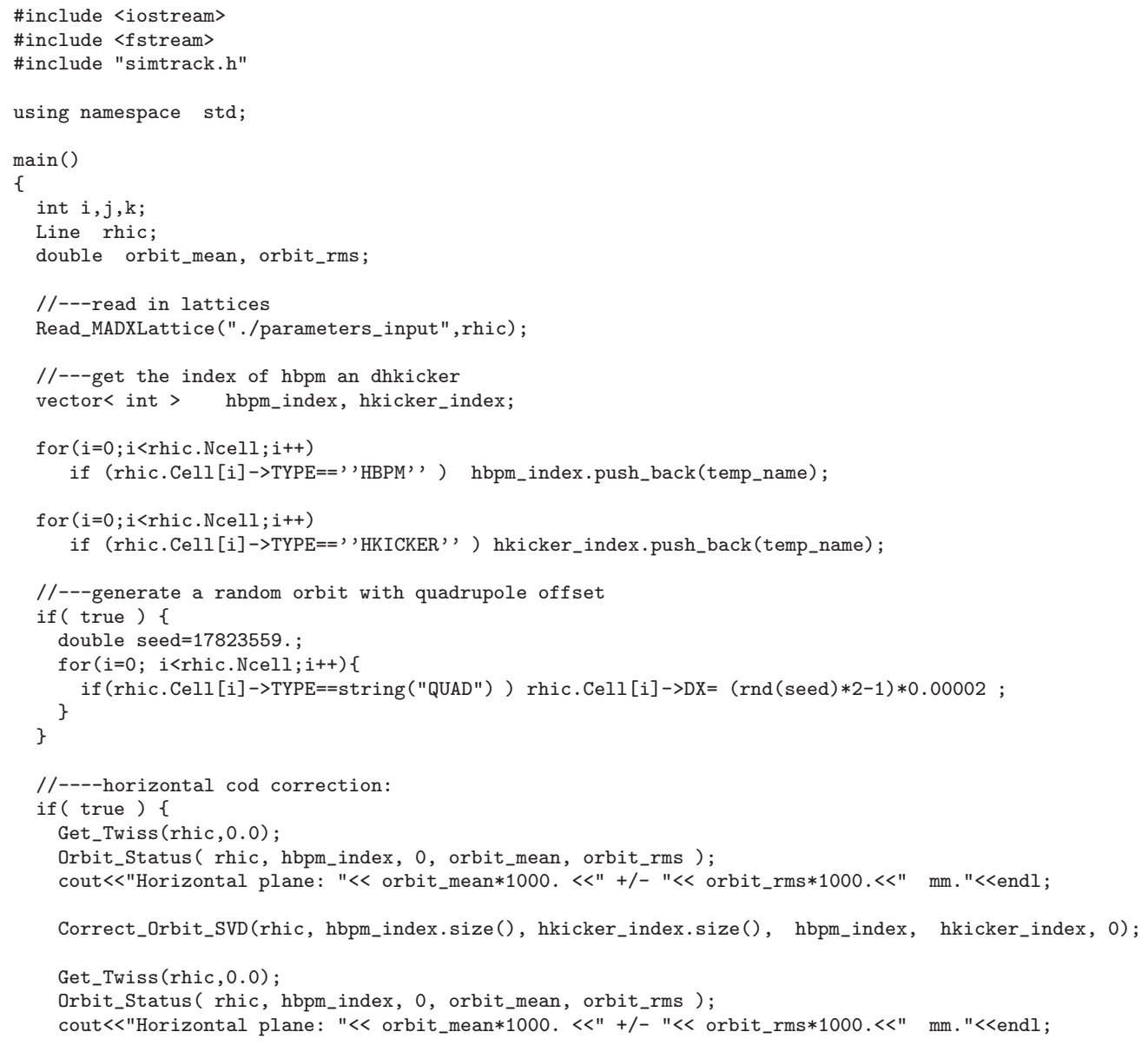




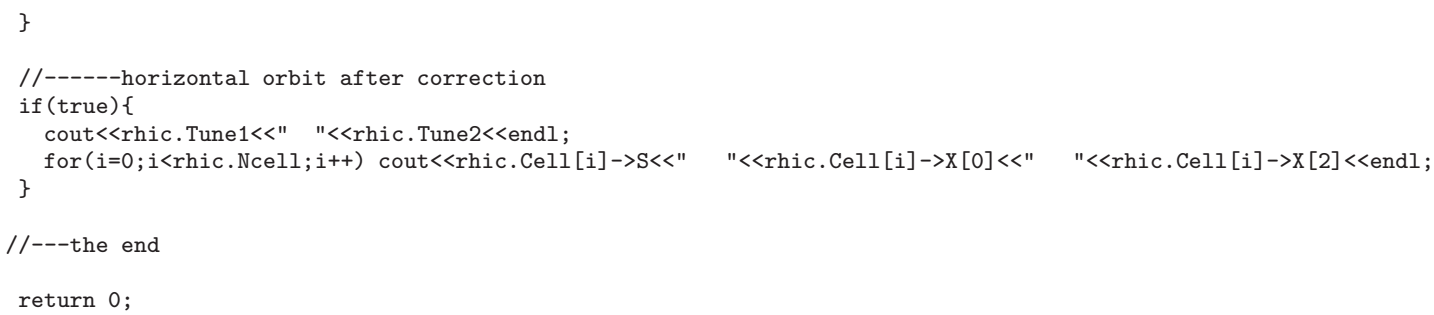

\section{Tracking}

\subsection{Track with Pass ()}

SimTrack supplies several conventional tracking functions,

void Track(Line \& linename, double x[6], int nturn, int \& stable, int \& lost_turn, int \& lost_post)

void Track_tbt(Line \& linename, double $x[6]$, int nturn, double x_tbt[], int \& stable, int \& lost_turn, int \& lost_post)

void Track_tbt_last100(Line \& linename, double x[6], int nturn, double x_tbt[], int \& stable, int \& lost_turn, int \& lost_post)

They all use function Pass(x[6]) in the tracking. The physical apertures of each element are used to determine if the particle is lost or not. The definitions of the input parameters are:

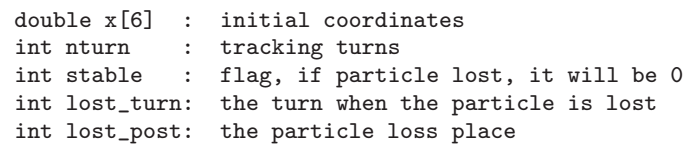

For example, let us look into the function Track(),

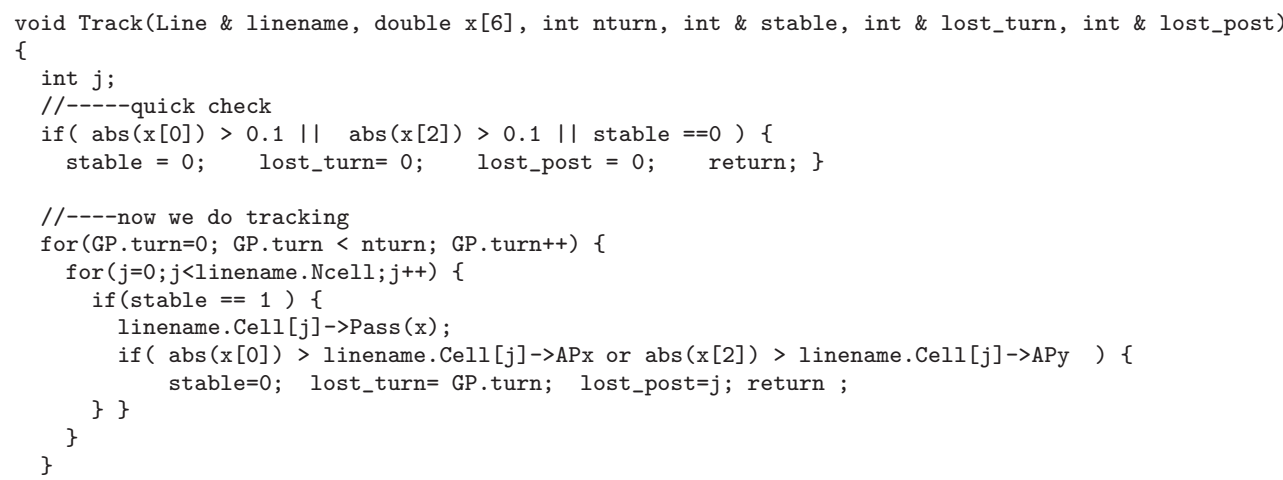

\subsection{Fast tracking without class}

To improve tracking speed, SimTrack also supplies some functions to allow track particles without c ++ class,

void Prepare_Track_Fast (Line \& linename)

void Track_Fast(Line \& linename, double x[6], int nturn, int \& stable, int \& lost_turn, int \& lost_post )

Before running Prepare_Track_Fast(), we normally first run the following two functions,

void MakeThin(Line \& linename)

void Concat_Drift(Line \& linename)

MakeThin() makes nonlinear elements to be zero length with drift-kick-drift model. Concat_Drift() concatenates the adjacent DRIFT elements. MakeThin() must be run before Prepare_Track_Fast() since Prepare_Track_Fast() treat MULT as zero length kick.

Function Prepare_Track_Fast() extracts and saves all lattice information and save them in some global variables which can will be used in function Track_Fast() use. For speed consideration, Track_fast() currently only supports the following types of elements: DRIFT, SBEND, QUAD, SEXT, MULT, RFCAV, BEAMBEAM, ELENS, ACMULT, COOLING, MATRIX. Other types of elements can be easily added into the fast tracking, too.

Following is an example for a long-term tracking, 


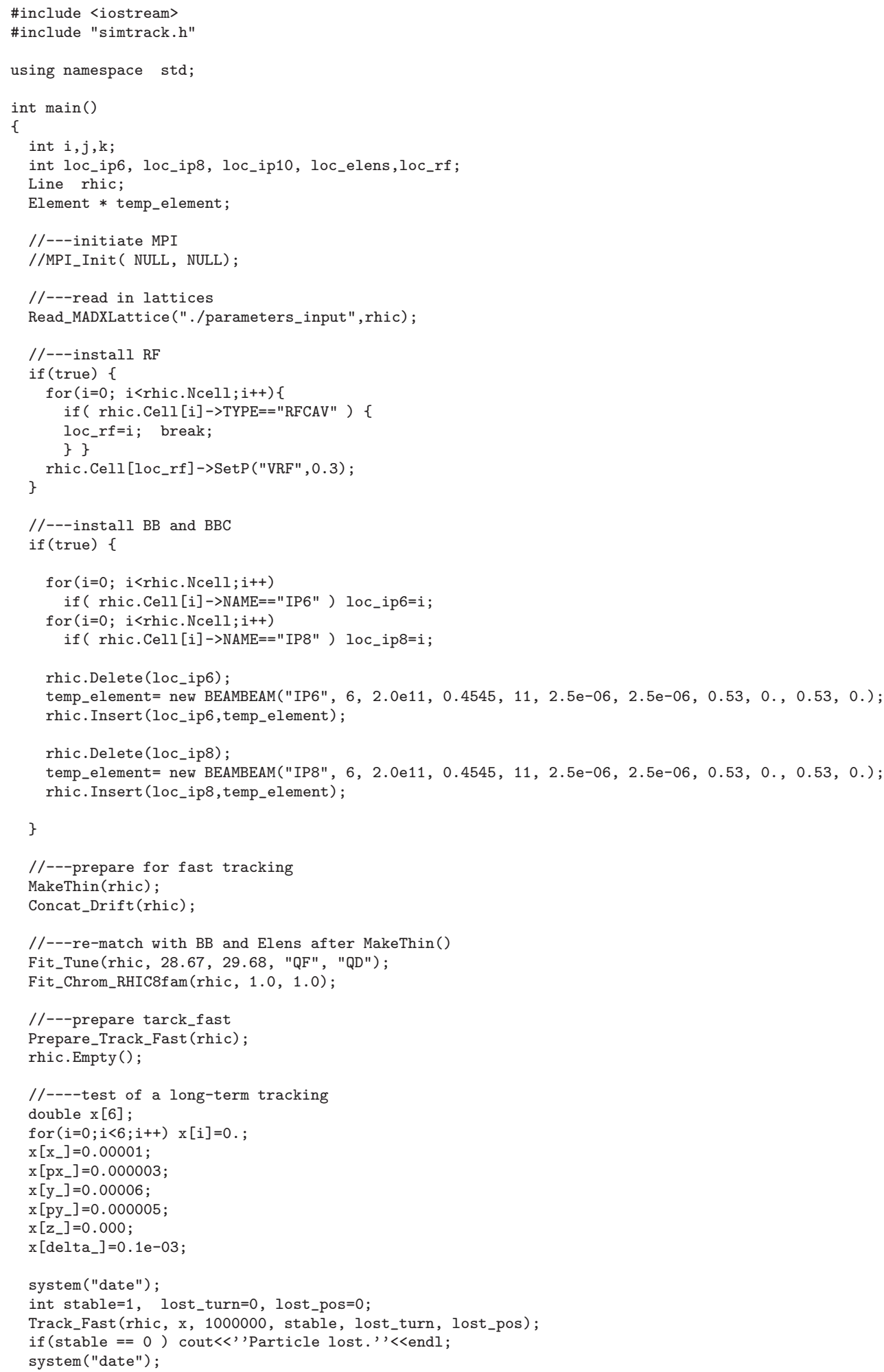

\section{Other Supporting Functions}

SimTrack also supplies some mathematics tools, like SVD, fine tune finder,etc.

long int fac(int $\mathrm{n}$ )

double rnd (double \& $r$ )

double guass (double u, double $g$, double \& $r$ )

void mat_mult(double $A[]$, double $B[]$, double $C[]$, int $m$, int $n$, int $k$ ) 
double mat_det(double a[], int $\mathrm{n}$ )

int mat_inv(double a [] , int $n$ )

void LinearEquations (double a1, double b1, double $\mathrm{c} 1$, double $\mathrm{a} 2$, double b2, double $\mathrm{c} 2$, double \& $\mathrm{x}$, double \& $\mathrm{y}$

void EigenSolver(double Matrix [4] [4], double wr [4], double wi[4], double vr[4] [4], double vi [4] [4])

int bmuav(double a [], int $\mathrm{m}$, int $\mathrm{n}$, double $*$, double $* \mathrm{v}$, double eps, int ka) // SVD method

void fft(int $\mathrm{m}$, double*x, double*y)

void Sin2FFT(int $n$, double*xr, double*xi, int \& nfft, double*famp, double*fphi)

void FineTuneFinder(int nfft, int $\mathrm{m}$, double $* x$, double \& fpeak)

SimTrack also supplies function to calculate frequency map, dynamic apertures and so on.

void Track_tbt_FMA( Line \& linename, double deltap0, double sigmax0, double sigmay0)

void Track_tbt_Lyapunov( Line \& linename, double deltap0, double sigmax0, double sigmayo)

void Track_tbt_last100_ActionDiff( Line \& linename, double deltap0, double exn0, double eyn0)

void Track_Fast_DA( Line \& linename, int nturn, double delta, double sigmax0, double sigmay0)

Following is an example to calculate frequency map with SimTrack library.

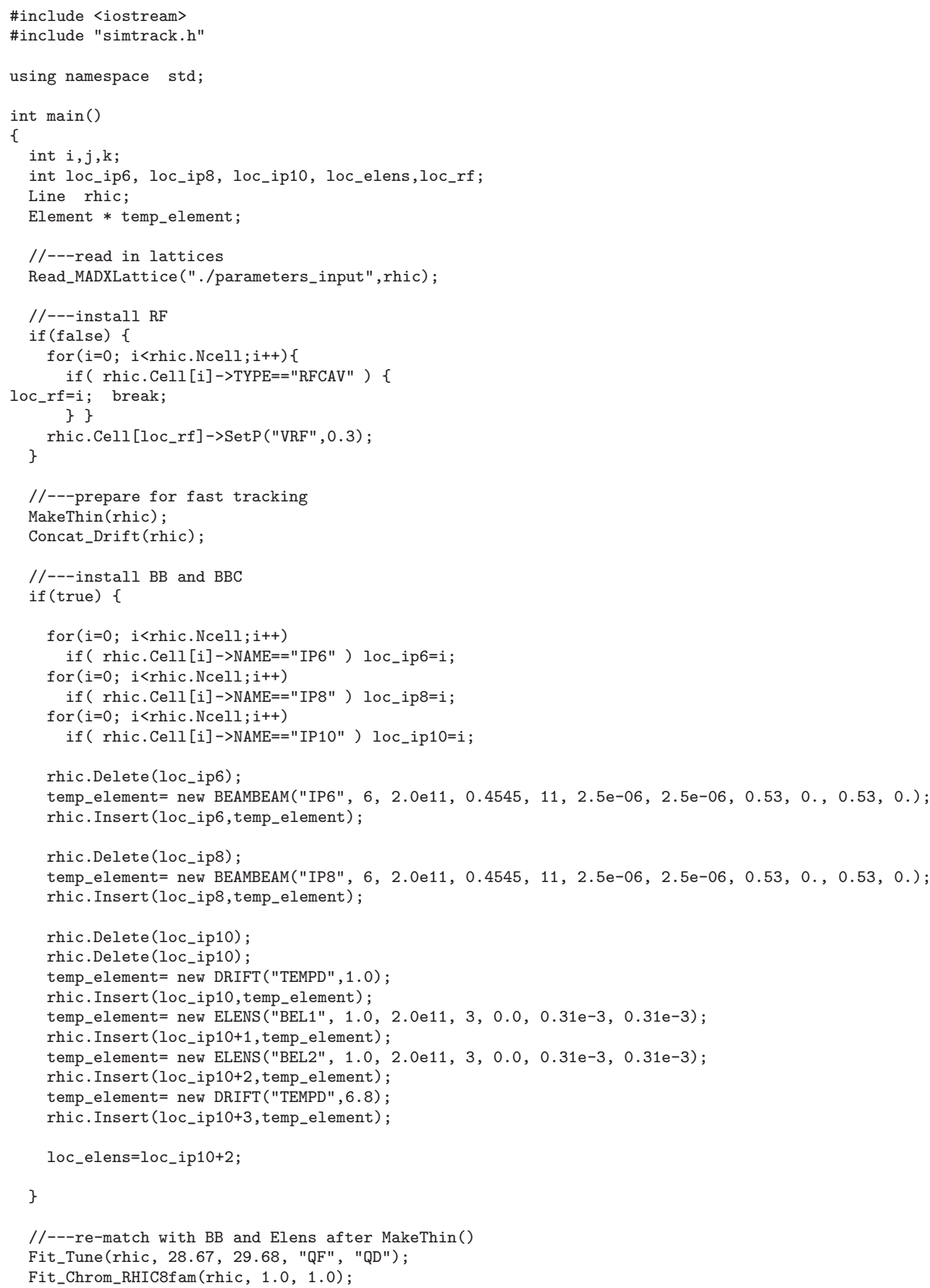


//---check the FMA

Track_tbt_FMA ( rhic, $0.0,0.06855 e-03,0.06855 e-03)$;

//--the end

return(0);

\}

Following is an example to calculate dynamic aperture with SimTrack library.

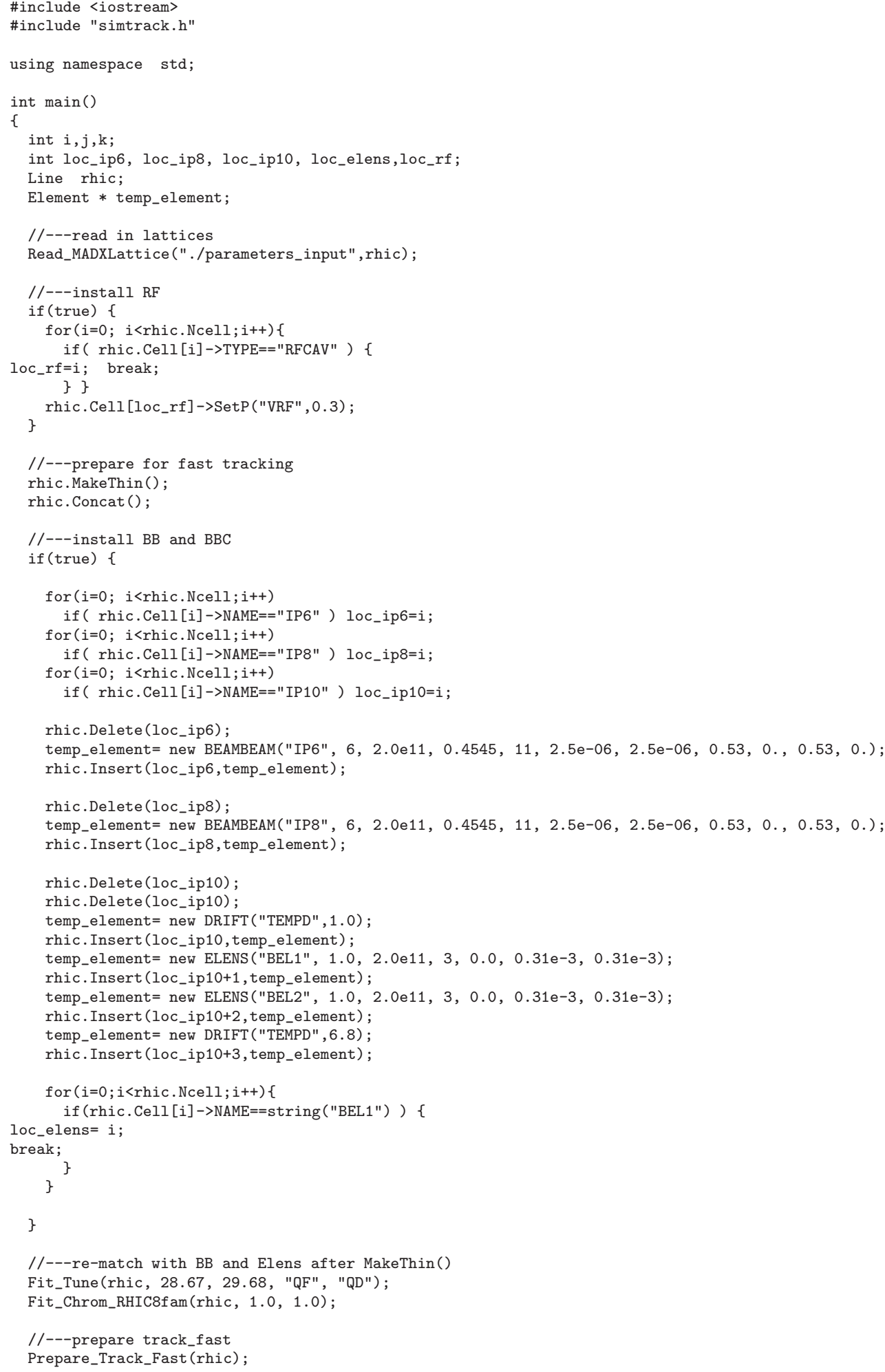


rhic.Empty();

//----DA tracking

int nturn=1000000;

double delta $=0.0005$;

double sigmax $0=\operatorname{sqrt}(2.5 \mathrm{e}-06 * 0.53 / 266$.$) ;$

double sigmay $0=\operatorname{sqrt}(2.5 e-06 * 0.53 / 266$.$) ;$

Track_Fast_DA ( rhic, nturn, delta, sigmax0, sigmay0);

//--the end

return(0);

\}

\section{Expansion: Define a New Type of Element}

To define a new type of element in SimTrack is straight-forward. You need define this type's specific parameters and its construction function, $\operatorname{GetP}(), \operatorname{SetP}()$ and $\operatorname{Pass}(), \operatorname{DAPass}()$. As an example, following is the block to define a RF cavity type of element in simtrack.h.

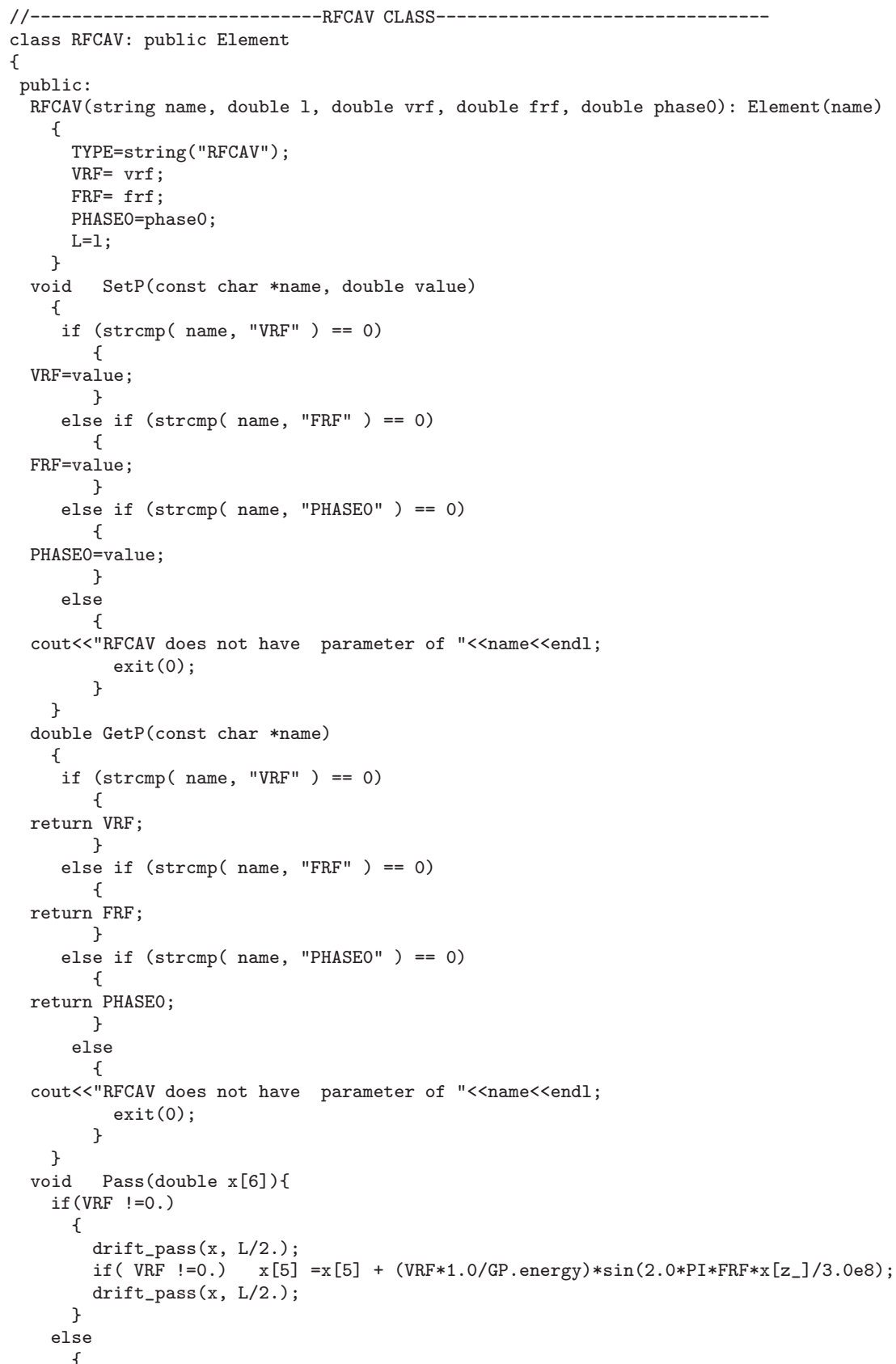




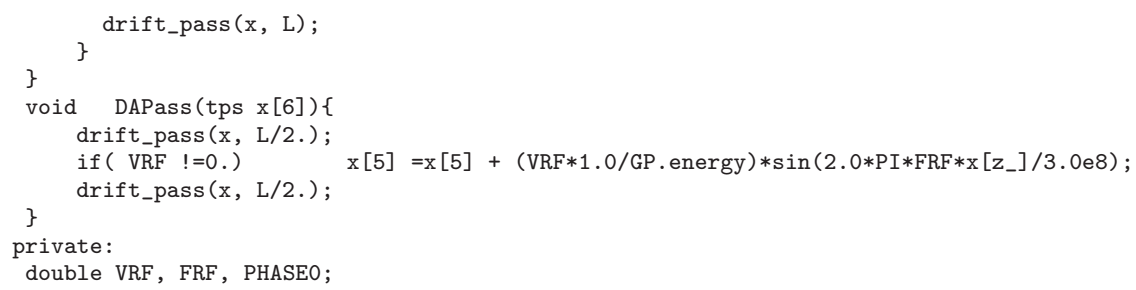

\section{Acknowledgement}

The author would like to thank Hyung Jin Kim for cross check of the 6-D weak-strong beam-beam treatment in SimTrack library.

\section{References}

[1] R.D. Ruth, "A canonical Integration Technique", IEEE Trans. Nucl. Sci., vol. NS-30, PP.2669-2671 (1983).

[2] J. Bengtsson, "The Sextupole Scheme for Swiss Light Source (SLS): An Analytic Approach", SLS Note 9/97, March 1997.

[3] M. Bassetti and G.A. Erskine, Closed expression for the electricalfield of a two-dimensional Gaussian charge, CERN-ISR-TH/80-06.

[4] K. Hirata, H. Moshammer, F. Ruggiero, A symplectic beam-beam interaction with energy change, Particle Accel. 40 (1993) 205-228.

[5] Y. Luo, Phys. Rev. ST Accel. Beam 7, 124001 (2004). 